\title{
On the Convergence of Central Path and Generalized Proximal Point Method for Symmetric Cone Linear Programming
}

\author{
Zhensheng Yu*,Yanyan Zhu and Qianqian Cao \\ College of Science, University of Shanghai for Science and Technology, Shanghai, 200093, P.R.China
}

Received: 21 Feb. 2013, Revised: 19 Jun. 2013, Accepted: 20 Jun. 2013

Published online: 1 Nov. 2013

Abstract: In this paper, we consider the symmetric cone linear programming(SCLP), by using the Jordan-algebraic technique, we extend the generalized proximal point method in linear programming and semidefinite programming to the SCLP. Under some reasonable conditions, we obtain the convergence of primal central paths associated to the symmetric cone distance function.

Keywords: Symmetric cone linear programming, Central path, Distance-like function, Generalized proximal point method.

\section{Introduction}

Let $\mathbb{A}=(\mathbb{V}, \circ,\langle\cdot, \cdot\rangle)$ be a Euclidean Jordan algebra(see Section 2 for the definition), where $(\mathbb{V},\langle\cdot, \cdot\rangle)$ is a finite dimensional inner product space over the real field $\mathbb{R}$ and "o" denotes the Jordan product which will be defined in the next section. Let $\mathscr{K}$ be the symmetric cone in $\mathbb{V}$ and denote $x \succeq_{\mathscr{K}} 0$ for $x \in \mathscr{K}$, we consider the symmetric cone linear programming (SCLP) with the primal form:

$$
\text { (P) } \min c^{T} x \text {, s.t. } \mathscr{A} x=b, x \succeq_{\mathscr{K}} 0,
$$

where the data consist of $c \in \mathbb{R}^{n}, \quad b \in \mathbb{R}^{m}$ and a linear operator $\mathscr{A}: \mathbb{V} \rightarrow \mathbb{R}^{m}$, the primal variable is $x \in \mathbb{V}$. The associated dual problem to $(\mathrm{P})$ is

$$
\text { (D) } \max b^{T} y \text {, s.t. } \mathscr{A}^{*} y+s=c, s \succeq \mathscr{K} 0,
$$

where $\mathscr{A}^{*}: \mathbb{R}^{m} \rightarrow \mathbb{V}$ denotes the adjoint application associated to $\mathscr{A}$ and $(s, y) \in \mathbb{V} \times \mathbb{R}^{m}$ are the dual variables. We denote the primal and dual feasible sets as $\mathscr{F}(P)=\left\{\begin{array}{lll}x \in \mathbb{V}: \mathscr{A} x=b, x \succeq_{\mathscr{K}} & 0\end{array}\right\} \quad$ and $\mathscr{F}(D)=\left\{(s, y) \in \mathbb{V} \times \mathbb{R}^{m}: \mathscr{A}^{*} y+s=c, s \succeq \mathscr{K} 0\right\}$, respectively. The interior of primal and dual feasible sets are denoted by $\mathscr{F}^{\circ}(P)=\left\{x \in \mathbb{V}: \mathscr{A} x=b, x \succ_{\mathscr{K}} 0\right\}$ and $\mathscr{F}^{\circ}(D)=\left\{(s, y) \in \mathbb{V} \times \mathbb{R}^{m}: \mathscr{A}^{*} y+s=c, s \succ \mathscr{K} 0\right\}$, and the primal and dual optimal sets are denoted by $\mathscr{F}^{*}(P)$ and $\mathscr{F}^{*}(D)$.

Throughout this paper, we assume that the following two conditions hold in the statements of our results.
A1) $\mathscr{A}: \mathbb{V} \rightarrow \mathbb{R}^{m}$ is a surjective linear operator;

A2) $\mathscr{F}^{0}(P) \neq \varnothing$ and $\mathscr{F}^{0}(D) \neq \varnothing$.

Assumption A1 is convenient to ensure that the dual variables $s$ and $y$ are in one-to-one correspondence. Assumption A2 ensures that both (P) and (D) have optimal solutions, the optimal values are equals and its solutions sets are bounded.

Nesterov and Todd[1] first proposed this optimization problem under the name of convex programming for self-scaled cones, and established polynomial complexity of primal-dual interior-point method applied to this problem using the so-called NT direction. It is well known that symmetric cone programming (SCP) includes linear programming (LP), semidefinite programming (SDP) and second order cone programming (SOCP) as special cases. Thus, some efficient methods in LP and SDP have been extended to the programming problems over symmetric cones. Faybusovich [2] first extended primal-dual IPMs to SCP through Euclidean Jordan algebraic tools. Schmieta and Alizadeh [3] proved polynomial iteration complexities for variants of the short-, semi-long-, and long-step path-following algorithms over symmetric cones. Vieira[4], [5] proposed primal-dual IPMs for SCP based on the kernel functions. Recently, Wang and Bai [6] generalized Darvays full-Newton step primal-dual path-following IPM for LP in [7] and presented a new full NT step primal-dual

\footnotetext{
*Corresponding author e-mail: zhsh-yu@ 163.com
} 
path-following IPM for SCP. Liu et al. [8] and Zhang and Zhang [9] proposed IPMs with the second-order corrector step for SCP and showed the polynomial convergence.

In this paper, we consider extending the well known interior point method-generalized proximal point method in LP (Linear Programming) and SDP (Semidefinite Programming) to the SCLP. This idea has, at first, appeared in Iusem et al. [10], they have proved this connection among central path and generalized proximal point sequence in some special cases, including linear programming. On the other hand, Doljansky and Teboule [11] introduced a generalized proximal method for unconstrained convex SDP problems and established its convergence properties. Recently, the method was extended to SDP by Ferreira, Oliveira and Silva [12] and Several works dealing with this issue include Aulender and Teboulle [13] and Mosheyev and Zibulevski [14]. More recently, Chen and Pan [15] considered an entropy-like proximal algorithm for convex symmetric cone programming in the form:

$$
\min f(x), \text { s.t. } x \succeq \mathscr{K} 0,
$$

where $f: V \rightarrow(-\infty,+\infty]$ is a closed proper convex function.

The main motivation of this paper is to develop the convergence of primal central paths associated to the symmetric cone distance functions for symmetric cone programming. Furthermore, we consider the generalized proximal point method with the distance function and establish the convergence of primal sequence.

The organization of this paper is as follows. In Sec.2, we review some basic concepts and materials on Euclidean Jordan algebra which are needed in the analysis of the generalized proximal point methods. In Sec.3, we list some good properties of the distance-like function in the symmetric cone $\mathscr{K}$. Furthermore, the detailed introduction and convergence analysis of the generalized proximal methods are given in Sec. 4

\section{Preliminaries on Euclidean Jordan algebra}

This section recalls some concepts and results on Euclidean Jordan algebras that will be used in the subsequent sections. More detailed expositions of Euclidean Jordan algebras can be found in Koecher's lecture notes [16] and Faraut and Korányi's monograph [17].

Let $\mathbb{V}$ be an $n$-dimensional vector space over the field of real numbers endowed with a bilinear mapping $(x, y) \rightarrow$ $x \circ y: \mathbb{V} \times \mathbb{V}$. For a given $x \in \mathbb{V}$, let $\mathscr{L}(x)$ be the linear operator of $\mathbb{V}$ defined by

$$
\mathscr{L}(x) y:=x \circ y \text { for every } y \in \mathbb{V} .
$$

The pair $(\mathbb{V}, \circ)$ is called a Jordan algebra if, for all $x, y \in$ $\mathbb{V}$, it holds that: (i) $x \circ y=y \circ x$,

(ii) $x \circ\left(x^{2} \circ y\right)=x^{2} \circ(x \circ y)$, where $x^{2}:=x \circ x$.

In a Jordan algebra $(\mathbb{V}, \circ), x \circ y$ is said to be the Jordan product of $x$ and $y$. Note that a Jordan algebra is not associative, i.e., $x \circ(y \circ z)=(x \circ y) \circ z$ may not hold in general. If for some element $e \in \mathbb{V}, x \circ e=e \circ x=x$ for all $x \in \mathbb{V}$, then $e$ is called a unit element of the Jordan algebra $(\mathbb{V}, \circ)$. For $x \in \mathbb{V}$, let $\zeta(x)$ be the degree of the minimal polynomial of $x$, which can be equivalently defined as

$\zeta(x):=\min \left\{k:\left\{e, x, x^{2}, \cdots, x^{k}\right\}\right.$ are linearly dependent $\}$.

Then the rank of $(\mathbb{V}, \circ)$, with a unit element $e \in \mathbb{V}$, defined over the real field $\mathbb{R}$ is called a Euclidean Jordan algebra or formally real Jordan algebra, if there exists a positive definite symmetric bilinear form on $\mathbb{V}$ which is associative; in other words, there exists on $\mathbb{V}$ an inner product denoted by $\langle\cdot, \cdot\rangle_{\mathbb{V}}$ such that for all $x, y, z \in \mathbb{V}$ :

(iii) $\langle x \circ y, z\rangle_{\mathbb{V}}=\langle y, x \circ z\rangle_{\mathbb{V}}$.

In a Euclidean Jordan algebra $\mathbb{A}=\left(\mathbb{V}, \circ,\langle\cdot, \cdot\rangle_{\mathbb{V}}\right)$, we define the set of squares as

$$
\mathscr{K}:=\left\{x^{2}: x \in \mathbb{V}\right\} .
$$

By [17], $\mathscr{K}$ is a symmetric cone. This means that $\mathscr{K}$ is a self-dual closed convex cone with nonempty interior and for any two elements $x, y \in \operatorname{int}(\mathscr{K})$, there exists an invertible linear transformation $\mathscr{T}: \mathbb{V} \rightarrow \mathbb{V}$ such that $\mathscr{T}(\mathscr{K})=\mathscr{K}$ and $\mathscr{T}(x)=y$.

Recall that an element $c \in \mathbb{V}$ is said to be idempotent if $c^{2}=c$. Two idempotents $c$ and $q$ are said to be orthogonal if $c \circ q=0$. So $\left\{c^{1}, c^{2}, \cdots c^{k}\right\}$ is a complete system of orthogonal idempotents if

$$
c_{j}^{2}=c_{j}, c_{j} \circ c_{i}=0 \text { if } j \neq i \text { for all } i, j=1,2, \ldots, k,
$$

and

$$
\sum_{k=1}^{k} c_{j}=e .
$$

An idempotent is said to be primitive if it is nonzero and cannot be written as the sum of two other nonzero idempotents. We call a complete system of orthogonal primitive idempotents a Jordan frame. Then we have the following spectral decomposition theorem.

2.1. [[11], Theorem III. 1.2] Suppose that $\mathbb{A}=\left(\mathbb{V}, \circ,\langle\cdot, \cdot\rangle_{\mathbb{V}}\right)$ is a Euclidean Jordan algebra and the rank of $\mathbb{A}$ is $r$. Then for any $x \in \mathbb{V}$, there exists a Jordan frame $\left\{c_{1}, c_{2}, \ldots, c_{r}\right\}$ and real numbers $\lambda_{1}(x), \lambda_{2}(x), \ldots, \lambda_{r}(x)$, arranged in the decreasing order $\lambda_{1}(x) \geq \lambda_{2}(x) \geq \ldots \geq \lambda_{r}(x)$, such that $x=\sum_{j=1}^{r} \lambda_{j}(x) c_{j}$. The numbers $\lambda_{j}(x)$ (counting multiplicities), which are uniquely determined by $x$, are called the eigenvalues; $\sum_{j=1}^{r} \lambda_{j}(x) c_{j}$ the spectral decomposition of $x$, and $\operatorname{tr}(x)=\sum_{j=1}^{r} \lambda_{j}(x)$ the trace of $x$. 


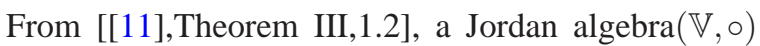
with a unit element $e \in \mathbb{V}$ is Euclidean if and only if the symmetric bilinear form $\operatorname{tr}(x \circ y)$ is positive definite. Therefore, we may define another inner product on $\mathbb{V}$ by

$$
\langle x, y\rangle:=\operatorname{tr}(x \circ y) \quad \text { for all } x, y \in \mathbb{V} .
$$

By the associativity of $\operatorname{tr}(\cdot)$ [[11], Proposition II.4.3], the inner product $\langle\cdot, \cdot\rangle$ is associative, i.e., for all $x, y, z \in \mathbb{V}$, there holds that $\langle x, y \circ z\rangle=\langle y, x \circ z\rangle$. Thus, the operator $\mathscr{L}(x)$ for each $x \in \mathbb{V}$ is symmetric with respect to the inner product $\langle\cdot, \cdot\rangle$ in the sense that

$$
\langle\mathscr{L}(x) y, z\rangle=\langle y, \mathscr{L}(x) z\rangle \forall y, z \in \mathbb{V} .
$$

Let $\|\cdot\|$ be the norm on $\mathbb{V}$ induced by the inner product $\langle\cdot, \cdot\rangle$, i.e.,

$$
\|x\|:=\sqrt{\langle x, x\rangle}=\left(\sum_{j=1}^{r} \lambda_{j}^{2}(x)\right)^{1 / 2} \quad \forall x \in \mathbb{V}
$$

and denote by $\lambda_{\min }(\cdot)$ and $\lambda_{\max }(\cdot)$ the smallest and the largest eigenvalue of $x$, respectively.

Let $g: \mathbb{R} \rightarrow \mathbb{R}$ be a scalar-valued function. Then, it is natural to define a vector-valued function associated with the Euclidean Jordan algebra $\left(\mathbb{V}, \circ,\langle\cdot, \cdot\rangle_{\mathbb{V}}\right)$ by

$$
g^{s c}:=g\left(\lambda_{1}(x)\right) c_{1}+g\left(\lambda_{2}(x)\right) c_{2}+\cdots+g\left(\lambda_{r}(x)\right) c_{r},
$$

where $x \in \mathbb{V}$ has the spectral decomposition $x=\sum_{j=1}^{r} \lambda_{j}(x) c_{j}$. This function is called the Löwner operator [15] and was shown to have the following important property.

Lemma2.1. [[18], Theorem13] For any $x=\sum_{j=1}^{r} \lambda_{j}(x) c_{j}$, let $g^{s c}$ be defined by (1). Then $g^{s c}$ is (continuously) differentiable at $x$ if and only if $g$ is (continuously) differentiable at all $\lambda_{j}(x)$. Furthermore, the derivative of $g^{s c}$ at $x$, for any $h \in \mathbb{V}$, is given by

$$
\begin{aligned}
\left(g^{s c}\right)^{\prime}(x) h= & \sum_{j=1}^{r} g^{\prime}\left(\lambda_{j}(x)\right)\left\langle c_{j}, h\right\rangle c_{j} \\
& +\sum_{1 \leq j<l \leq r} 4\left[\lambda_{j}(x), \lambda_{l}(x)\right]_{g} c_{j} \circ\left(c_{l} \circ h\right)
\end{aligned}
$$

with

$$
\left[\lambda_{j}(x), \lambda_{l}(x)\right]_{g}:=\left(g\left(\lambda_{j}(x)\right)-g\left(\lambda_{l}(x)\right)\right) /\left(\lambda_{j}(x)-\lambda_{l}(x)\right)
$$

$$
\forall j, l=1,2, \ldots, r \text { and } j \neq l .
$$

In particular, we introduce the inverse function

$$
\begin{aligned}
& x^{-1}=\lambda_{1}^{-1}(x) c_{1}+\lambda_{2}^{-1}(x) c_{2}+\cdots+\lambda_{r}^{-1}(x) c_{r}, \\
& \quad \text { for all } \lambda_{i}(x) \neq 0 .
\end{aligned}
$$

\section{Properties of Distance-like Function}

To design the algorithm, we introduce the distance-like function as follows (see [15]):

$$
\begin{gathered}
H(x, y):=\operatorname{tr}(x \circ \ln x-x \circ \ln y+y-x), \\
\forall x \in \operatorname{int}(\mathscr{K}), y \in \operatorname{int}(\mathscr{K})
\end{gathered}
$$

Adding $H\left(x, x^{0}\right)$ in the objective function of $(\mathrm{P})$, we obtain its penalized version

$\left(P_{\mu}\right) \min c^{T} x+\mu H\left(x, x^{0}\right)$ s.t. $\mathscr{A} x=b, x \succ_{\mathscr{K}} 0, \mu>0$.

where $x \succ \mathscr{K} 0$ means that $x \in \operatorname{int}(\mathscr{K}), x^{0} \succ \mathscr{K} 0$ is a given point.

So the primal central path to the problem $(\mathrm{P})$, with respect to the function $H\left(x, x^{0}\right)$ is the set of points $\{x(\mu): \mu>0\}$. where $x(\mu)$ is defined as

$$
(x(\mu))=\arg \min _{x \succ \mathscr{K} 0}\left\{c^{T} x+\mu H\left(x, x^{0}\right): \mathscr{A} x=b\right\}, \mu>0 .
$$

For the discussion of next section, we propose the favorable properties of the distance measure $\mathrm{H}$, and the following two technical lemmas will be used, the first lemma is given by M.Baes [19].

Lemma3.1 For any $x, \quad y \in \mathbb{V}$, we have $\operatorname{tr}(x \circ y) \leq \sum_{j=1}^{r} \lambda_{j}(x) \lambda_{j}(y)=\lambda(x)^{T} \lambda(y)$, where $\lambda(x)$ and $\lambda(y)$ are the spectral vectors of $x$ and $y$, respectively.

Lemma3.2[[15], Lemma 3.2] For any $x \in \operatorname{int}(\mathscr{K})$, let $\Phi(x):=\operatorname{tr}(x \circ \ln x)$. Then, we have the following results.

(i) $\Phi(x)$ is the spectral function generated by the symmetric entropy function

$$
\phi(u)=\sum_{j=1}^{r} u_{j} \ln u_{j} \quad \forall u \in \mathbb{R}_{+}^{r} .
$$

(ii) $\Phi(x)$ is continuously differentiable on $\operatorname{int}(\mathscr{K})$ with $\nabla \Phi(x)=\ln x+e$.

(iii) The function $\Phi(x)$ is strictly convex over $\mathscr{K}$.

In lemma 3.2, we can know that the vector-valued function $x \circ \ln x$ is the Löwner function $g^{s c}(x)$, i.e., $g^{s c}=x \circ \ln x$. Clearly, $g^{s c}$ is well-defined for any $x \in \mathscr{K}$ and

$$
g^{s c}(x)=x \circ \ln x=\sum_{j=1}^{r} \lambda_{j}(x) \ln \left(\lambda_{j}(x)\right) c_{j}
$$

Therefore,

$\Phi(x)=\operatorname{tr}(x \circ \ln x)=\operatorname{tr}\left(g^{s c}(x)\right)=\sum_{j=1}^{r} \lambda_{j}(x) \ln \left(\lambda_{j}(x)\right)=\phi(\lambda(x))$.

Next we introduce the properties of the distance-like function $\mathrm{H}$. These properties play a crucial role in the convergence analysis of the central path in the following 
section, the proof can be found in [12].

Lemma3.3 Let $H(x, y)$ be defined by (4). Then the following results hold.

(i) $H(x, y)$ is continuous on $\mathscr{K} \times \operatorname{int}(\mathscr{K})$ and $H(\cdot, y)$ is strictly convex for any $y \in \operatorname{int}(\mathscr{K})$.

(ii) For any fixed $y \in \operatorname{int}(\mathscr{K}), H(x, y)$ is continuously differentiable on $\operatorname{int}(\mathscr{K})$ with

$$
\nabla_{x} H(x, y)=\ln x-\ln y .
$$

(iii) $H(x, y) \geq 0$ for any $x \in \mathscr{K}$ and $y \in \operatorname{int}(\mathscr{K})$, and $H(x, y)=0$ if and only if $x=y$.

(iv) For fixed $y \in \operatorname{int}(\mathscr{K})$, the level sets $L_{H}(x, \gamma):=\{x \in \mathscr{K} \mid H(x, y) \leq \gamma\}$ are bounded for all $\gamma \geq 0$.

\section{Generalized Proximal Point Method}

From(6), we know that $x(\mu)$ is the solution of the problem $\left(P_{\mu}\right)$. For the convergence analysis of the generalized proximal methods, we first introduce the following theorem.

Lemma4.1 The primal central path defined by (6) is well defined and is in $\mathscr{F}^{0}(P)$.

Proof. For any $\mu>0$ we define $\Psi_{\mu}: \operatorname{int}(\mathscr{K}) \rightarrow \mathbb{R}$ by

$$
\Psi_{\mu}(x)=c^{T} x+\mu \operatorname{tr}\left(x \circ \ln x-x \circ \ln x^{0}+x^{0}-x\right) .
$$

From Lemma3.3(i), we know that the function $\Psi_{\mu}(x)$ is strictly convex and extends continuously to $\mathscr{K}$ with the convention that $0 \circ \ln 0=0$. Its gradient is given by $\nabla \Psi_{\mu}(x)=c+\mu \ln (x)-\mu \ln \left(x^{0}\right)$ by using Lemma3.3(ii) and the unique minimizer is $e^{-c / \mu+\ln \left(x^{0}\right)}$.

Take $\tilde{x} \in \mathscr{F}^{0}(P)$, from Lemma3.3(iv) we know that $\mathscr{L}=\left\{x \in \mathscr{K} \mid \Psi_{\mu}(x) \leq \Psi_{\mu}(\tilde{x})\right\}$ is bounded and nonempty. Since $\Psi_{\mu}(x)$ is continuous in $\mathscr{K}$, we get that $\mathscr{L}$ is compact and nonempty, since $\mathscr{F}(P)$ is closed and nonempty, we have that $\mathscr{L} \cap \mathscr{F}(P)$ is also compact and nonempty. Therefore, the strictly convexity of $\Psi_{\mu}(x)$ implies that it has a unique minimizer. Thus the primal central path is well defined.

Next it remains to show that $x(\mu) \in \mathscr{F}^{0}(P)$. Assume by contradiction that $x(\mu) \in \partial \mathscr{F}(P)=\{x \succeq \mathscr{K} 0, \mathscr{A} x=$ $b, x \in b d(\mathscr{K})\}$. Define

$$
z_{\varepsilon}=(1-\varepsilon) x(\mu)+\varepsilon \tilde{x}
$$

where $\varepsilon \in(0,1)$. Then, as $\tilde{x} \in \mathscr{F}^{0}(P), x(\mu) \in \partial \mathscr{F}(P)$, $\varepsilon \in(0,1)$ and $\mathscr{F}^{0}(P)$ is convex, we conclude that $z_{\varepsilon} \in \mathscr{F}^{0}(P)$ for all $\varepsilon \in(0,1)$. Now combining definitions of $x(\mu)$ and $z_{\varepsilon}$ with convexity of $\Psi_{\mu}(\cdot)$ after some algebraic manipulation we obtain

$$
\begin{aligned}
0 \leq \Psi_{\mu}\left(z_{\varepsilon}\right)-\Psi_{\mu}(x(\mu)) & \leq\left\langle\nabla \Psi_{\mu}\left(z_{\varepsilon}\right), z_{\varepsilon}-x(\mu)\right\rangle \\
& =\varepsilon /(1-\varepsilon)\left\langle\nabla \Psi_{\mu}\left(z_{\varepsilon}\right), \tilde{x}-z_{\varepsilon}\right\rangle
\end{aligned}
$$

which implies $0 \leq\left\langle\nabla \Psi_{\mu}\left(z_{\varepsilon}\right), \tilde{x}-z_{\varepsilon}\right\rangle$, so from Lemma3.1, we get

$$
\begin{aligned}
0 & \leq\left\langle\nabla \Psi_{\mu}\left(z_{\varepsilon}\right), \tilde{x}-z_{\varepsilon}\right\rangle \\
& =\left\langle c+\mu \ln \left(z_{\varepsilon}\right)-\mu \ln \left(x^{0}\right), \tilde{x}-z_{\varepsilon}\right\rangle \\
& =\mu \operatorname{tr}\left(\tilde{x} \circ \ln \left(z_{\varepsilon}\right)-c^{T} z_{\varepsilon}-\mu \operatorname{tr}\left(z_{\varepsilon} \circ \ln \left(z_{\varepsilon}\right)\right)\right. \\
& \left.-\mu \operatorname{tr}\left(\tilde{x} \circ \ln \left(x^{0}\right)\right)+\mu \operatorname{tr}\left(z_{\varepsilon}\right) \circ \ln \left(x^{0}\right)\right)+c^{T} \tilde{x} \\
& \leq \mu \sum_{i=1}^{r} \lambda_{i}(\tilde{x}) \lambda_{i}\left(\ln \left(z_{\varepsilon}\right)\right)-\Psi_{\mu}\left(z_{\varepsilon}\right)+c^{T} \tilde{x} \\
& -\mu \operatorname{tr}\left(z_{\varepsilon}-x^{0}+\tilde{x} \circ \ln \left(x^{0}\right)\right)+c^{T} \tilde{x}
\end{aligned}
$$

Under the hypothesis $x(\mu) \in \partial \mathscr{F}(P)$, using the fact that $z_{\varepsilon}$ goes to $x(\mu)$ as $\varepsilon$ goes to $0, \tilde{x} \succ \mathscr{K} 0$ and as the function $\Psi_{\mu}$ is continuous, the right side of the above inequality goes to $-\infty$. Therefore, we get an absurd which implies the desired result.

Lemma 4.1 guarantees that the primal central path to the Problem $(\mathrm{P})$, with respect to the function $H\left(\cdot, x^{0}\right)$, is well defined and is in $\mathrm{F} 0(\mathrm{P})$. So, for all $\mu>0$, we have from Eq.(6) that

$$
c+\mu\left(\ln (x(\mu))-\ln \left(x^{0}\right)\right)=\mathscr{A}^{*} y(\mu),
$$

for some $y(\mu) \in \mathbb{R}^{m}$.

The dual central path associated to the problem $(\mathrm{P})$ is the set of points $\{s(\mu): \mu>0\}$, where $s(\mu)$ satisfies

$$
s(\mu)=-\mu\left(\ln (x(\mu))-\ln \left(x^{0}\right)\right), \quad \mu>0,
$$

or equivalently, $(s(\mu), y(\mu))$ is the unique solution of the optimization problem

$$
\max \left\{b^{T} y-\mu t r e^{-s / \mu+\ln \left(x^{0}\right)}: \mathscr{A}^{*} y+s=c\right\}, \quad \mu>0 .
$$

Thus the set $\{(x(\mu), y(\mu), s(\mu)): \mu>0\}$ denotes the primal-dual central path and it is the unique solution of the following system

$$
\begin{aligned}
& \mathscr{A} x=b, \quad x \succ \mathscr{K} 0, \\
& \mathscr{A}^{*} y+s=c, \\
& s+\mu \ln (x)-\mu \ln \left(x^{0}\right)=0, \quad \mu>0 .
\end{aligned}
$$

This completes the proof.

Lemma4.2 The primal-dual central path is an analytic curve contained in $\operatorname{int}(\mathscr{K}) \times \mathbb{R} \times \mathscr{K}$.

Proof. First we introduce the map $\Upsilon: \operatorname{int}(\mathscr{K}) \times \mathbb{R}^{m} \times \mathscr{K} \times$ $\mathbb{R}_{++} \rightarrow \mathbb{R}^{m} \times \mathscr{K} \times \mathscr{K}$ given by

$$
\Upsilon(x, y, s, \mu)=\left(\begin{array}{c}
\mathscr{A} x-b \\
\mathscr{A}^{*} y+s-c \\
\mu \ln (x)-\mu \ln \left(x^{0}\right)+s
\end{array}\right) .
$$

Note that $Y(x, y, s, \mu)=0$ is equivalent to the system Eq.(10). Since the central path is the unique solution of the system Eq.(10) we have that

$$
\Upsilon(x(\mu), y(\mu), s(\mu), \mu)=0, \text { forall } \mu>0 .
$$


So, as $\Upsilon$ is an analytic function the statement follows from the implicit function theorem by showing that its Jacobian with respect to $(x, y, s)$ is nonsingular everywhere. To show that the Jacobian of $\Upsilon$ is nonsingular it is sufficient to prove that its null-space is the trivial one. Assume that

$$
\nabla_{(x, y, s)} \Upsilon(x, y, s, \mu)(u, v, w)=0
$$

equivalently,

$$
\begin{aligned}
& \mathscr{A} u=0, \\
& \mathscr{A}^{*} v+w=0, \\
& \mu x^{-1} \circ u+w=0 .
\end{aligned}
$$

Last equation of Eq.(11) implies that $w=-\mu x^{-1} \circ u$. Substituting in the second equation of Eq.(11) we get $\mu u=x \circ \mathscr{A}^{*} v$ and in view of the first equation $\mathscr{A}\left(x \circ \mathscr{A}^{*} v\right)=0$. Finally as $x \succ \mathscr{K} 0$ and $\mathscr{A}$ is surjective we have that $\mathscr{A} \mathscr{L}(x) \mathscr{A}^{*}$ is nonsingular, thus latter equality implies that $v=0$ and consequently $w=u=0$. Therefore, the Jacobian of $\Upsilon$ is nonsingular and the statement follows.

Lemma4.1 The following statements hold

(i) the function $0<\mu \mapsto H\left(x(\mu), x^{0}\right)$ is non-increasing,

(ii) the set $\{x(\mu): 0<\mu \leq \bar{\mu}\}$ is bounded, for each $\bar{\mu}$,

(iii) all cluster points of the primal central path are solutions of the problem $(\mathrm{P})$.

Proof. (i) For all $\mu>0$, we know that the equation of (8) is equivalent to

$$
\left.\mu \nabla_{x} H\left(x(\mu), x^{0}\right)\right)=-c+\mathscr{A}^{*} y(\mu) .
$$

for some $y(\mu) \in \mathbb{R}^{m}$.

Take $\mu_{1}, \mu_{2}>0$ with $\mu_{1}<\mu_{2}$, since $H$ is convex and $x\left(\mu_{1}\right)-x\left(\mu_{2}\right) \in$ Null $\mathscr{A}$ we have from the previous equality that

$$
\begin{aligned}
& \mu_{1}\left(H\left(x\left(\mu_{1}\right), x^{0}\right)-H\left(x\left(\mu_{2}\right), x^{0}\right)\right) \\
& \left.\leq \mu_{1}\left\langle\nabla_{x} H\left(x\left(\mu_{1}\right), x^{0}\right)\right), x\left(\mu_{1}\right)-x\left(\mu_{2}\right)\right\rangle \\
& =-c^{T}\left(x\left(\mu_{1}\right)-x\left(\mu_{2}\right)\right)
\end{aligned}
$$

and

$$
\begin{aligned}
& \mu_{2}\left(H\left(x\left(\mu_{2}\right), x^{0}\right)-H\left(x\left(\mu_{1}\right), x^{0}\right)\right) \\
& \left.\leq \mu_{2}\left\langle\nabla_{x} H\left(x\left(\mu_{2}\right), x^{0}\right)\right), x\left(\mu_{2}\right)-x\left(\mu_{1}\right)\right\rangle \\
& =-c^{T}\left(x\left(\mu_{2}\right)-x\left(\mu_{1}\right)\right) .
\end{aligned}
$$

Now combining the latter two equations we obtain that $\left(\mu_{1}-\mu_{2}\right)\left(H\left(x\left(\mu_{1}\right), x^{0}\right)-H\left(x\left(\mu_{2}\right), x^{0}\right)\right) \leq 0$ and as $\mu_{1}<\mu_{2}$ we have that $H\left(x\left(\mu_{1}\right), x^{0}\right) \geq H\left(x\left(\mu_{2}\right), x^{0}\right)$. So the statement (i) is established.

(ii) Now fixed $\bar{\mu}>0$. Similar argument used to prove item (i) implies that

$$
\mu\left(H\left(x(\mu), x^{0}\right)-H\left(x(\bar{\mu}), x^{0}\right)\right) \leq-c^{T}(x(\mu)-x(\bar{\mu}))
$$

for all $0<\mu \leq \bar{\mu}$. From item (i) we have that $0 \leq H\left(x(\mu), x^{0}\right)-H\left(x(\bar{\mu}), x^{0}\right)$ for all $0<\mu \leq \bar{\mu}$. Then above equation implies that $c^{T} x(\mu) \leq c^{T} x(\overline{\bar{\mu}})$, for all $0<\mu \leq \bar{\mu}$. So

$$
\{x(\mu): 0<\mu \leq \bar{\mu}\} \subset\left\{x \in \mathscr{F}(P): c^{T} x \leq c^{T} x(\bar{\mu})\right\} .
$$

Since $\mathscr{F}^{*}(P)$ is bounded we have that the sublevel $\left\{x \in \mathscr{F}(P): c^{T} x \leq c^{T} x(\bar{\mu})\right\}$ is also bounded. Therefore the statement (ii) follows from the last inclusion.

(iii) Let $\bar{x}$ be a cluster point of $\{x(\mu): \mu>0\}$. First note that $\mathscr{A} \bar{x}=b$, and $\bar{x} \succeq \mathscr{K} 0$, i.e. $\bar{x} \in \mathscr{F}(P)$. Let $\left\{\mu_{k}\right\}$ be a sequence of positive numbers such that $\lim _{k \rightarrow+\infty} \mu_{k}=0$, and $\lim _{k \rightarrow+\infty} x\left(\mu_{k}\right)=\bar{x}$. Take $x^{*}$ a solution of $(\mathrm{P})$ and $x \in$ $\mathscr{F}^{0}(P)$. For $\varepsilon>0$, define

$$
y(\varepsilon)=(1-\varepsilon) x^{*}+\varepsilon x
$$

Due the fact that $x^{*} \in \partial \mathscr{F}^{0}(P), x \in \mathscr{F}^{0}(P)$, for $\varepsilon \in(0,1]$, from (6), we have

$$
c^{T} x\left(\mu_{k}\right)+\mu_{k} H\left(x\left(\mu_{k}\right), x^{0}\right) \leq c^{T} y(\varepsilon)+\mu_{k} H\left(y(\varepsilon), x^{0}\right),
$$

or,

$$
\mu_{k}\left(H\left(x\left(\mu_{k}\right), x^{0}\right)-H\left(y(\varepsilon), x^{0}\right)\right) \leq c^{T}\left(y(\varepsilon)-x\left(\mu_{k}\right)\right) .
$$

Now since $H\left(\cdot, x^{0}\right)$ is convex and $y(\varepsilon) \in \mathscr{F}^{0}(P)$, it is easy to conclude from above inequality that

$$
\mu_{k}\left\langle\nabla_{x} H\left(y(\varepsilon), x^{0}\right), x\left(\mu_{k}\right)-y(\varepsilon)\right\rangle \leq c^{T}\left(y(\varepsilon)-x\left(\mu_{k}\right)\right)
$$

Thus taking limits in the latter inequality as $k \rightarrow+\infty$. we obtain $0 \leq c^{T}(y(\varepsilon)-\bar{x})$, in this inequality, if $\varepsilon \rightarrow 0$, it gives $0 \leq c^{T}\left(x^{*}-\bar{x}\right)$ or $c^{T} \bar{x} \leq c^{T} x^{*}$. The statement (iii) is proved.

Theorem4.1 Let $\hat{x} \in \mathscr{K}$ be the analytic center of $\mathscr{F}^{*}(P)$, i.e.,there exists the unique point satisfying

$$
\hat{x}=\arg \min \left\{H\left(x, x^{0}\right): x \in \mathscr{F}^{*}(P)\right\}
$$

then $\lim _{\mu \rightarrow 0} x(\mu)=\hat{x}$.

Proof. From Lemma3.3(iv), it is easy to see that $H\left(\cdot, x^{0}\right)$ is continuous in $\mathscr{K}$, with the convention $0 \circ \ln 0=0$. Take $\bar{x}$ a cluster point of the primal central path and $\left\{\mu_{k}\right\}$ be a sequence of positive numbers such that $\lim _{k \rightarrow+\infty} \mu_{k}=0$, and $\lim _{k \rightarrow+\infty} x\left(\mu_{k}\right)=\bar{x}$. Now from Eq.(6), we have $\mu \nabla_{x} H\left(x\left(\mu_{k}\right), x^{0}\right)=-c+\mathscr{A}^{*} y\left(\mu_{k}\right)$, for some $y\left(\mu_{k}\right) \in \mathbb{R}^{m}$. So,

$\mu_{k}\left\langle\nabla_{x} H\left(x\left(\mu_{k}\right), x^{0}\right), x-x\left(\mu_{k}\right)\right\rangle=\left\langle-c+\mathscr{A}^{*} y\left(\mu_{k}\right), x-x\left(\mu_{k}\right)\right\rangle$, for all $x \in \mathscr{F}^{*}(P)$, using the convexity of $H$ and the fact that $x-x\left(\mu_{k}\right) \in$ Null $\mathscr{A}$, the latter equation becomes

$$
\mu_{k}\left(H\left(x\left(\mu_{k}\right), x^{0}\right)-H\left(x, x^{0}\right)\right) \leq c^{T} x-c^{T} x\left(\mu_{k}\right) .
$$

Since $x \in \mathscr{F}^{*}(P)$ and $\mu_{k}>0$, it follows from the latter inequality that $H\left(x\left(\mu_{k}\right), x^{0}\right) \leq H\left(x, x^{0}\right)$. Now as $H$ is continuous we can take limits, as $k \rightarrow+\infty$ in this inequality to conclude that $H\left(\bar{x}, x^{0}\right) \leq H\left(x, x^{0}\right)$ for all $x \in \mathscr{F}^{*}(P)$. Thus any cluster point of the primal central path satisfies Eq.(12). Therefore, since $\hat{x}$ is the unique point satisfying Eq.(12), the primal central path converges to it and the theorem is proved. 


\section{Final Remarks}

In this paper we have studied the convergence of primal central paths associated to the for SCLP problems. The asymptotic behavior of the primal and dual trajectories associated to the distance-like function in linear program and semidefinite programming has been studied by Cominetti and San Martłn[20] and Ferreira[12]. We do not have considered the behavior of the primal-dual sequence in the generalized proximal method. Since, we do not know how to prove the convergence of the primal-dual sequence to the solution, and so we leave it as an open problem.

\section{Acknowledgement}

This work are supported by National Natural Science Foundation of China (No.11171221) and National Project and Liberal Base Cultivate Fund of USST(No.12GXM)

\section{References}

[1] Y. E. Nesterov, M. J. Todd, Primal-dual interior-point methods for self-scaled cones, Technical Report 1125, School of Operations Research and Industrial Engineering, Cornell University, Ithaca, New York, (1995).

[2] L. Faybusovich, Linear systems in Jordan algebras and primalCdual interior-point algorithms. J. Comput. Appl. Math, 86, 149-175 (1997).

[3] S. H. Schmieta, F. Alizadeh, Extension of primalCdual interior point algorithm to symmetric cones. Math. Program, 96, 409-438 (2003).

[4] M. V. C. Vieira, Jordan algebras approach to symmetric optimization. Ph.D. thesis, Delft University of Technology, (2007).

[5] M. V. C.Vieira, Interior-point methods based on kernel functions for symmetric optimization. Optim. Methods Softw, 27, 513-537 (2012).

[6] G. Q. Wang, Y. Q. Bai, A new full NesterovCTodd step primalCdual path-following interior-point algorithm for symmetric optimization. J. Optim. Theory Appl, 154, 966985 (2012).

[7] Z. Darvay, New interior-point algorithms in linear programming. Adv. Model. Optim, 5, 51-92 (2003).

[8] C. H. Liu, H. W. Liu, X. Z. Liu, Polynomial convergence of second-order Mehrotra-type predictor corrector algorithms over symmetric cones. J. Optim. Theory Appl., 154, 949-965 (2012).

[9] J. Zhang, K. Zhang, Polynomial complexity of an interior point algorithm with a second order corrector step for symmetric cone programming. Math. Methods Oper. Res, 73, 75-90 (2011).

[10] A. N. Iusem, B. F. Svaiter, J. X. da Cruz Neto. Central Paths, Generalized Proximal Point Methods, and Cauchy Trajectories in Riemannian Manifolds, SIAM J. Cont. Optim, 37, 566-588 (1999).
[11] M. Doljansky, M. Teboulle. An Interior Proximal Algorithm and the Exponential Multiplier Method for Semidefinite Programming. SIAM J. Optim, 9, 1-13 (1998).

[12] O. P. Ferreira, P. R. Oliveira, R. C. Silva. On the convergence of the entropy-exponential penalty trajectories and generalized proximal point methods in semidefinite optimization. J. Global. Optim, 45, 211-227 (2009).

[13] A. Aulender, M.Teboulle, Interior gradient and proximal methods for convex and conic optimization. SIAM J. Optim, 16, 697-725 (2006).

[14] L. Mosheyev, M.Zibulevski, Penalty/barrier multiplier algorithm for semidefinite programming. Optim. Methods Softw, 13, 235-261 (2000).

[15] J. S. Chen, S. H. Pan. An entropy-like proximal algorithm and the exponential multiplier method for convex symmetric cone programming, Comput. Optim. Appl, 47, 477-499 (2010).

[16] M. Koecher, The Minnesota Notes on Jordan Algebras and Their Applications, edited and annotated by A. Brieg and S. Walcher, Springer, Berlin, (1999).

[17] J. Faraut and A. Korányi, Analysis on Symmetric Cones, Oxford Mathematical Monographs, Oxford University Press, New York, (1994).

[18] D. Sun, J. Sun, Löwner operator and spectral functions on Euclidean Jordan algebras, Math. Oper. Res, 33, 421-445 (2008).

[19] M. Baes, Convexity and differentiability properties of spectral functions and spectral mappings on Euclidean Jordan algebras. Linear. Alg. Appl, 422, 664-700 (2007).

[20] R. Cominetti, J. San Martłn, Asymptotic analysis of the exponential penalty trajectory in linear programming. Math. Program, 67, 169-187 (1994). 


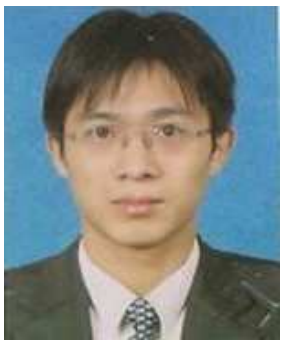

Zhensheng Yu received the $\mathrm{PhD}$ degree in Operations Research and Control Theory at Dalian University of Technology. His research interests are in the areas of optimization algorithms and its applications, nonlinear complementarity theory and methods. He has published research articles in reputed international journals of mathematical and applications mathematics.

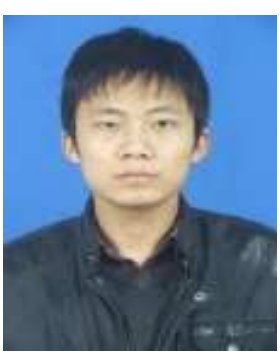

Yanyan Zhu received the Ms degree in applied mathematics at University of Shanghai for Science and Technology. His research interests are in the areas of optimization algorithms and its applications, nonlinear complementarity theory and methods.

Qianqian Cao received the Ms degree in applied mathematics at University of Shanghai for Science and Technology. Her research interests are in the areas of optimization algorithms and its applications, nonlinear complementarity theory and methods. She has published research articles in reputed international journals of mathematical and applications mathematics. 